\title{
SS433: THE MICROQUASAR LINK WITH ULXS?
}

\author{
P. A. Charles, ${ }^{1}$ A. D. Barnes, ${ }^{1}$ J. Casares,${ }^{2}$ J. S. Clark,${ }^{3}$ W. I. Clarkson, ${ }^{1}$ E. T. Harlaftis, ${ }^{4}$ R. I. Hynes, ${ }^{5}$ \\ T. R. Marsh, ${ }^{1}$ and D. Steeghs ${ }^{6}$
}

RESUNEX

\begin{abstract}
$\$ \$ 433$ es el microcuatsar prototipo de la Galaxia y posiblemente incluso análogo a las fuentes ULX si se tiene cn cuenta la "nergía cincitica do los "jets". Sin embargo y a pesar de 20 años de estudio, nuestras restricciones solbr. la naturaleya del sistrua binario son "xtremalamente grandes debido a la dificultad de localizar rasgos cspectrales capaces de revelar la naturaleza y el movimiento del donador de la masa. Los espectros azules de alta resolución recirntemente obteniclos cuindo el disco (en precesión) se encuentra de perfil, sugieren que la binaria se halla cerca de la fisse de rnvolvente común, lo cual hace extremadamente difícil obtener las restricciones cinéticas. Siu ombargo, (nocontranos evidencia de un donador masivo como era de esperar para la muy alta

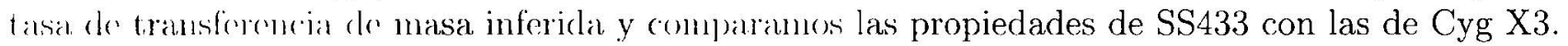

\section{ABSTRACT}

SS:13:3 is the prototype microquasar in the Galaxy and may even be analogous to the ULX sources if the jets' kinctic colergy is laken into acrount. However, in spite of 20 years of study, our constraints on the nature of the binary system are extremely limited as a result of the difficulty of locating spectral features that can reveal the nature and motion of the mass donor. Newly acquired, high resolution blue spectra taken when the (precessing) disc is edge-on sigggest that the binary is close to a common-envelope phase, and hence providing kinc matic constraints is extremely difficult. Nevertheless, we do find evidence for a massive donor, as expected for the inferred very high mass transfer rate, and we compare SS433's properties with those of Cyg X-3.

\section{liey Words: ACCRETION: ACCRETION DISCS - X-RAYS: BINARIES - X-RAYS: STARS}

\section{INTRODUCTION}

StS4:3:3 holds a special place in late 20 th century astronomy as the tirst relativistic jet somere discov(reel in the Gialaxy (see Margon 198.t), and hence is the prototype microquasar. It is remarkable as the only continuously emitting microquasar, with its kne foalure being the $162.5 \mathrm{~d}$ precession period of the jets (and assoriated accrolion disc) that is revealed b) the "moving lincs" which have now been observed for more than 2 decades. The radial velocity curves of the moving lines are very well described by the Kinematic Model (Margon 1984), therehy revaling key parameters of the jets $\left(v=0.26 c ; i=79^{\circ}\right)$ and, combined with radio observations of the associated $5 \mathrm{NR} 1150$. a distance of $4.8 \mathrm{kpc}$. It is observed as a woak $\left(\sim 10^{36}{ }^{36 g} s^{-1}\right)$ X-ray source (X1909+(0)+8), which combined with the high orbital inclination (it is eclipsing) suggests that it may be an Accretion Disc Corona (AD(') type of X-ray souree. How-

\footnotetext{
${ }^{1}$ iniversity of Southampton. Southampton. Uk

${ }^{2}$ Instituto de Astrofísica de Canarias, La Laguna, Spain.

${ }^{3}$ University College Iondon, Uk

${ }^{4}$ National Observatory, Athens. Greece

${ }^{5}$ Lniversity of Treats at Austin, IS.1

${ }^{6}$ Harvarrl-Smithsonian Conter for Astrophysics. Cambridge, I'SA.

ever, it is well established that the kinetic energy in the jets is at least 1,000 times greater than the observed luminosity (Safi-Harb 2003), although the origin of the jet energy is still a matter of debate, and this would place SS433 in the recently defined class of ULX sources (e.g. King 2003). If the intrinsic luminosity were indeed $\geq 10^{39} \mathrm{erg} \mathrm{s}^{-1}$, then the implied mass-transfer rate would be extremely high $\left(\dot{M} \sim 10^{-4} M \odot y^{-1}\right.$, see also Fuchs et al 2003).

This has led to King et al (2000) investigating the evolutionary status of SS433 and they show that, given a massive, radiative companion, it is possible for SS433 to avoid the common-envelope phase, by expelling the majority of the transferred material (part of which constitutes the jets). They conclude that the observed X-rays are then likely to almost entircly arise from the jets themselves. Indeed, the $\mathrm{X}$-ray output of SS433 appears to be dominated by the precessing jets, with moving X-ray emission lines and even spatially resolved $\mathrm{X}$-ray structure along the jet axis (Marshall et al 2002; Namiki et al 2003). Recently, however, the RXTE All-Sky Monitor has revealed a long-term modulation in the precessional $\mathrm{X}$-ray lightcurve (Gies et al 2001). The relativistic doppler boosting from jet precession alone is insuff- 


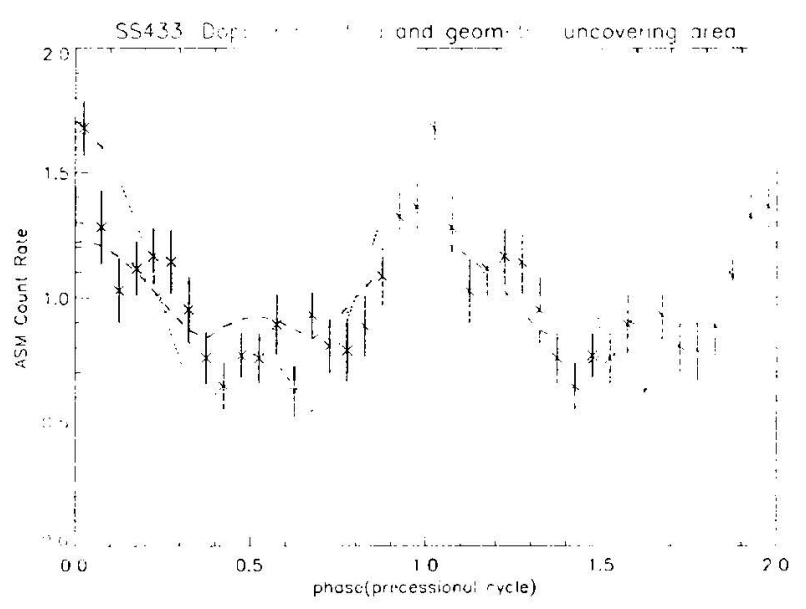

Fig. 1. 7-year X-ray lightcurve of SS 433 obtained with the RXTE/ASM. The dotted and dashed curves represent modulation expected from doppler boosting in the relativistic jets (for the cases of bullets and a continuous stream respectively). An additional geometric modulation is clearly required, modelled here as the modulation of a power-law jet by a precessing accretion disk (dotdashed line). From Clarkson et al (2004).

cient to produce this modulation, which requires an additional, presumed geometrical component (Clarkson et al 2004). For the established high inclination this can most naturally be provided by structure in the edge-on view of the accretion disk, which may be tilted or warped to produce the apparent asymmetry in the precessional lightcurve.

\section{DYNAMICAL CONSTRAINTS}

While the binary period (of 13d) is also wellknown, the nature of the mass donor $(\mathrm{a} \sim \mathrm{B}$ star; Crampton \& Hutchings 1981) is poorly constrained because of the high reddening in combination with strong disc/jet emission features that obliterate the characteristic (and weak) absorption lines of carlytype stars. Consequently, only emission line radial velocity curves have been derived with any confidence (Fabrika \& Bychkova 1990), which means that dynamical constraints on the system masses are limited. Indeed, with no evidence for X-ray pulsations or bursts, there is no obvious indication as to the nature of the compact object. And the interpretation of the radial velocity curves requires assumptions about. the mass donor, leading to estimates that range from $0.8 \mathrm{M}_{\odot}$ (d'Odorico et al 1991) to $62 \mathrm{M}_{\odot}$ (Antokhina \& Cherepashchuk 1985)!

Nevertheless Fabrika \& Bychkova used $\sim 4$ yrs of $6 \mathrm{~m}$ data taken around precession phase $\Psi \sim 0$ (which minimises the scatter in their radial velocity curve), corresponding to the most open viewing angle of the disc (and hence best view of the hotter inner dise regions). For an assumed $e=0$ they obtain
$f\left(M_{2}\right) \simeq 8 \mathrm{M}$. which gives $M_{2} \sim 10 \mathrm{M}$. for a $\mathrm{KS}$. or $15 M_{\odot}$ for a $6 M_{\odot} \mathrm{BH}$ (both are capable of explaining the extremely high $M$ ).

Consequently it has been impossible to add SS433 to the growing list of compact object mass determinations (Charles \& Coe 2003) which is so important for a system whose properties link it to both the microquasars and ULX systems.

\section{DETECTION OF MASS DONOR IN SS433?}

Recognising the noed to mestigate the nature of the mass donor if progress was to be made in understanding SS+33. it became clear to sereral groups that high $\mathrm{S} / \mathrm{N}$, high resolution blue spectra were crucial in order to search for weaker spertral features in absorption (away from the strong disc emission lines). Assuming that the best chance to search for the donor signature would come when the disc had precessed (with the jets) to its maximum opening angle, Girs et al (2002) obtained spectra that suggested weak absorption features similar to those seen in late A supergiants. These did yield a fow radial velocity measurements, but could only be used to derive mass constraints in combination with the HeII emission radial velocity curve (sce figure 3 ). from which they obtained $q=M_{\mathrm{X}} / M_{2}=0.6 \pm 0.1$, and $M_{X}=11 \pm 5 \mathrm{M} \ldots, M_{2}=19 \pm 7 \mathrm{M} \ldots$. Such an extreme mass ratio would be expected in order to explain the required very high (thermal) mass transfer rate (King et al 2000). However, their extremely limited orbital phase coverage clearly indicated the need for further observations to confirm and extend these results.

In fact, we had been acquiring such blue spectra of SS433 for several years (from AAT, WHT. INT. and Calar Alto), in an attempt to obtain good sampling in both orbital and precessional phases. Our initial aim was to search for any irradiation signature of the donor via the Bowen fluorescence mechanism, as has been so successful in Sco X-1 and other luminous X-ray binaries (see (aisares et al. these proceedings). Unfortunately, no such component wiss visible. only broad Bowen emission, likely from an extended region such as the clisc. We had also decided to take a different route to Gies ot al when seeking evidence for the donor itself, and therefore examined our spectra obtained around precessional phases $\sim 0.3-0 . \bar{T}$. when the jots are close to perpendicular to our lineof-sight, and correspondingly the accretion disc is seen close to edge-on. Since the strong optical variability as a function of precessional phase is already well-established (and indicates a disc contribution at least equal to that of the mass donor), we felt that 


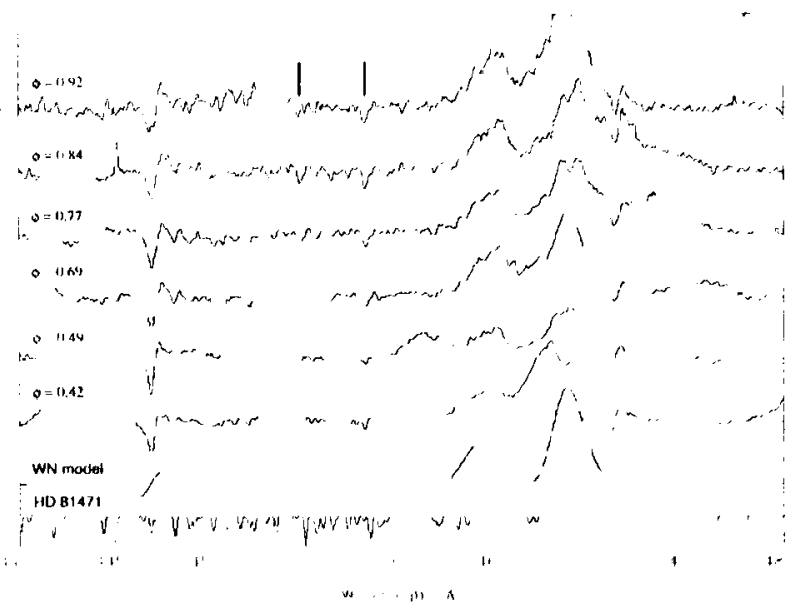

Fig. 2. Blue spectra of S\$4333 obtained near precession phase $\sim 0.5$, when the disc is closest to edge on. The sharp absorption features between $\lambda \lambda 4500-4600$ are characteristic of late B A spectral types and we associate these with the mass donor. The bottom two spectra show (upper) the similarity of the gross SS433 cmission features to that of a high mass-loss WN star and (lower) an A7I template star HD 81471.

it was important to minimise the disc component by observing when the disc was edge-on. Our best spectra obtained at these phases are shown in figure 2 . Of particular interest are the weak, but narrow, absorption features in the $\lambda \lambda 4500-4600$ region. These are mostly FeII transitions and are typically seen in early-type (late B - A) spectra. This includes the spectral type suggested by Gies et al, but we could not detect the transitions they reported. However, we have now clearly detected such features in SS433, and these are marked in figure 2, and are strong in the A7 template (HD81471) plotted at the bottom.

What was more remarkable was the radial velocity curve of these absorption features (see figure 3 ). While the velocity around orbital phase 0.75 is close to that expected from Gies et al's values for their mass ratio, the subsequent velocities (approaching phase 1.0) move in the opposite direction! This only makes sense if the secondary is embedded in a dense outflowing disc wind, although we might expect these lines to then show $\mathrm{P}$ Cyg behaviour under such circumstances, and the velocities around phase $0.4-0.5$ are even more anomalous. Nevertheless, with this orbital phase behaviour, it becomes extremely difficult to justify a keplerian interpretation of the observed velocities, and hence any resulting masses would be subject to major systematic effects. And support for the concept of a major disc plane (equatorial) mass outflow has already been obtained via the radio detection of extended emission in a direction that is perpendicular to the main jet outflow

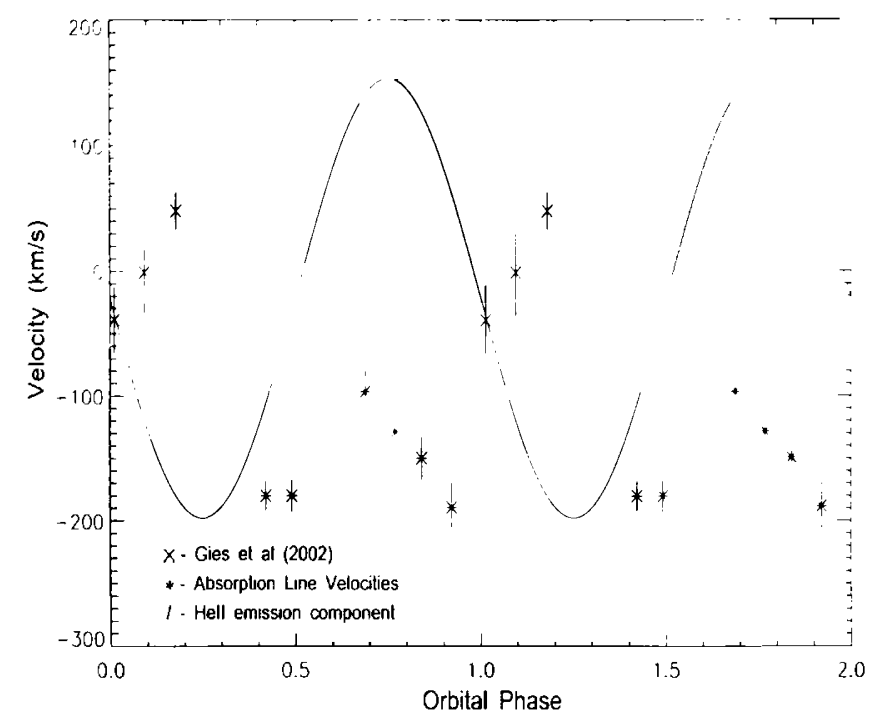

Fig. 3. Absorption line radial velocity measurements of SS433 obtained by us $\left(^{*}\right)$ and Gies et al $2002(\mathrm{x})$, together with the Fabrika \& Bychkova 1990 fit to their HeII emission velocities which were presumed to represent the: disc/compact object.

(Blundell et al 2001). Furthermore, the overall SS433 spectrum is remarkably similar to models of WN spectra (e.g. 2nd from bottom plot of figure 2 from Clark \& Porter 2004 which is a model for the WN9 star WR105 with parameters of $T=32,500 \mathrm{~K}, \dot{M}=$ $1.2 \times 10^{-5} M_{\odot} y^{-1}$ and $v_{\infty}=700 \mathrm{~km} \mathrm{~s}^{-1}$ ), and represents a dense outflow in which an ionising source is embedded. We are not claiming that SS433 is a W-R star, but simply point out that it makes little difference whether a hot star or powerful X-ray source is the source of that ionisation, both of which are of course present in Cyg X-3 (Fuchs et al 2003), which may even be the end result of the evolution of systems like SS433.

\section{REFERENCES}

Blundell, K.M. et al. 2001, ApJ, 562, L79

Casares, J. et al. 2003, ApJ, 590, 1041

Charles, P. \& Coe, M.J. 2003, astro-ph/0308020

Clark, J.S. \& Porter, J. 2004, A\&A submitted

Clarkson, W.I. et al. 2004 (in preparation)

Crampton, D. \& Hutchings, J.B. 1981, ApJ, 251, 604

Fabrika, S.N. \& Bychkova, L.V. 1990, A\&A, 240, L5

Fuchs, Y. et al. 2003, astro-ph/0208432

Gies, D.R. et al. 2002, ApJ, 578, L67

Hynes. R.I. et al. 2003, ApJ, 583, L95

King, A.R. 2003, astro-ph/0301118

Marshall, H.L. et al. 2002, ApJ, 564, 941

Namiki, MI. et al. 2003, PASJ, 55, 281

Safi-Harb, S. 2003, in New Views on Microquasars, p243

Steeghs, D. \& Casares, J. 2002, ApJ, 568, 273

Wade. R.A.. \& Horne, K. 1988, ApJ, 324, 411 